\title{
Effect of Reconstruction Parameters in High-Definition PET/CT on Assessment of Lymph Node Metastases in Head and Neck Squamous Cell Carcinoma
}

\author{
Maliha Sadick ${ }^{1}$, Flavia Molina ${ }^{2}$, Sabine Frey ${ }^{3}$, Rafael Piniol ${ }^{3}$, Haneen Sadick ${ }^{3}$, Joachim Brade ${ }^{4}$, Christian Fink ${ }^{5}$, \\ Stefan O. Schoenberg ${ }^{1}$, and Yong $\mathrm{He}^{6}$ \\ ${ }^{1}$ Institute of Clinical Radiology and Nuclear Medicine, University Hospital Mannheim, Medical Faculty Mannheim of the University \\ of Heidelberg, Mannheim, Germany; ${ }^{2}$ Clinic for Radiotherapy and Radiation Oncology, University Hospital Mannheim, Medical \\ Faculty Mannheim of the University of Heidelberg, Mannheim, Germany; ${ }^{3}$ Department of ENT and Head and Neck Surgery, \\ University Hospital Mannheim, Medical Faculty Mannheim of the University of Heidelberg, Mannheim, Germany; ${ }^{4}$ Department of \\ Biostatistics, University Hospital Mannheim, Medical Faculty Mannheim of the University of Heidelberg, Mannheim, Germany; \\ ${ }^{5}$ Department of Radiology, Allgemeines Krankenhaus Celle, Celle, Germany; and ${ }^{6}$ Department of Nuclear Medicine and PET Center, \\ Union Hospital, Tongji Medical College, Hua Zhong University of Science and Technology, Wuhan, People's Republic of China
}

Therapy outcome and overall survival in patients with head and neck squamous cell carcinoma (HNSCC) is influenced by precise localization of the primary tumor and detection of lymph node metastasis involvement at the time of initial diagnosis. Only accurate preoperative staging can improve primary tumor response and avoid early locoregional recurrence with lymph node metastases. The purpose of this study was the optimization of reconstruction parameters in high-definition PET/CT for the improved diagnostic assessment of lymph node metastases. Methods: In the experimental study, image contrast and signal-to-noise ratio were evaluated using a Jaszczak phantom. In the clinical study, 54 patients underwent head and neck imaging on a PET/CT scanner. Diagnostic findings were correlated with postoperative histopathology. For the 54 patients, 123 lymph nodes were evaluated on PET and histologically correlated with the neck dissection specimen. Forty-one lymph nodes were benign, and 82 findings were confirmed as being malignant. Both experimental and clinical studies were reconstructed into a $200 \times 200$ matrix using a 3-dimensional iterative reconstruction algorithm (ordered-subset expectation maximization [OSEM], 3 iterative steps, 24 subsets). Postfiltering with a 3-dimensional gaussian filter was applied. To study the effect of smoothing filter strength on the diagnostic accuracy of lymph node metastasis detection, 3 different cutoffs-1, 3, and $6 \mathrm{~mm}$ in full width at half maximum-were used to perform reconstructions. Results: Phantom studies showed that images reconstructed with 3-mm gaussian postfiltering gained a higher image quality and signal-to-noise ratio. Overall sensitivities for correctly diagnosed lymph nodes were best in 3-mm postfiltered images. Best results for true-positive lymph node findings were achieved with 3-mm postfiltering. With 1-mm postfiltering, accurate lesion detection was not improved, because increas-

Received Nov. 5, 2012; revision accepted Nov. 29, 2012

For correspondence or reprints contact: Maliha Sadick, Institute of Clinical Radiology and Nuclear Medicine, University Hospital Mannheim, Medical Faculty Mannheim of the University of Heidelberg, Theodor-Kutzer Ufer 1-3, 68167 Mannheim, Germany.

E-mail: maliha.sadick@umm.de

Published online Jan. 30, 2013.

COPYRIGHT @ 2013 by the Society of Nuclear Medicine and Molecular Imaging, Inc. ing sensitivity (95\% true-positive) correlated with decreasing specificity (12\% false-positive). Conclusion: For lymph node assessment on a high-resolution PET/CT scanner, we consider the OSEM algorithm with 3 iterations and 24 subsets, combined with 3-dimensional 3-mm gaussian postfiltering, to be optimal. The continuous application of presently established PET protocols in patients with HNSCC will prove whether current acquisition and reconstruction methods are valuable and should be maintained.

Key Words: head and neck squamous cell carcinoma; lymph node metastasis; ${ }^{18}$ F-FDG PET/CT; FWHM; gaussian filter

J Nucl Med Technol 2013; 41:19-25

DOI: 10.2967/jnmt.112.116806

D espite current imaging concepts that include CT, MR tomography, and PET/CT, diagnosis of early-stage head and neck squamous cell carcinoma (HNSCC) and involved lymph node metastases remains a challenge (1-3). Considering that this tumor entity represents the fifth most common cancer worldwide, with HNSCC being newly diagnosed in 500,000 people every year, only accurate preoperative staging can improve primary tumor response and locoregional control $(4,5)$. Therapeutic outcome and overall survival in patients with HNSCC very much depends on local primary tumor size, infiltration of adjacent vascular structures, and presence and spread of lymph node metastases at the time of diagnosis (6). ${ }^{18} \mathrm{~F}-\mathrm{FDG}$ PET/CT meanwhile is an established imaging modality for lymph node staging in HNSCC, achieving high overall diagnostic sensitivity, specificity, and accuracy of more than $90 \%(1,3,7,8)$.

Hybrid PET/CT delivers combined anatomic and functional information. It is part of the oncologic work-up of tumors with regard to diagnosing, staging, and evaluating therapy response (9). Especially in the head and neck region, with its small anatomic structures, special attention is 
required for optimal diagnostic throughput. In this setting, the selection of dedicated scanning protocols and image reconstruction parameters plays an important role (10). Iterative reconstruction techniques in combination with application of various spatial gaussian postfilters influence spatial resolution of PET images, image contrast, and signal-to-noise ratio (SNR) $(10,11)$.

The purpose of this study in patients with an initial diagnosis of HNSCC was the optimization of reconstruction parameters in high-definition (HD) PET/CT for improved diagnostic assessment of lymph node metastases. We also investigated the influence of parameter settings on image quality and on standardized uptake value (SUV) monitoring of suggestive cervical lymph nodes in HNSCC.

\section{MATERIALS AND METHODS}

\section{Phantom Study}

The imaging quality study was performed using a body phantom from the National Electrical Manufacturers Association (NEMA)/International Electrotechnical Commission with 6 fillable spheres, with a radius from 10 to $37 \mathrm{~mm}$ to simulate hot and cold lesions. A cylindric insert filled with low-atomic-number material $(0.3 \pm 0.1 \mathrm{~g} / \mathrm{mL})$ was used to simulate attenuation. The background activity concentration was $5 \mathrm{kBq} / \mathrm{mL}$, with a hot-tobackground ratio of $4: 1$, similar to those found in the head and neck region. To simulate the activity out of the scanner, a line source approximately $70 \mathrm{~cm}$ long with an activity of $109 \mathrm{MBq}$ was inserted in a large solid polyethylene cylinder. The data were acquired in list mode for 3 min per bed scan. The images were reconstructed using an ordered-subset expectation maximization (OSEM) algorithm with 3 iterations and 24 subsets. The following reconstruction parameters were used: a matrix size of $200 \times 200$ (pixel size, $4.07 \mathrm{~mm}$ ); zoom of 1; and gaussian filters of 1, 3, and 6 $\mathrm{mm}$ in full width at half maximum (FWHM). This procedure was performed 3 times in order to check the reproducibility of the results. Reconstructed images were analyzed with the image quality test following the NEMA protocol NU2-2007 (12). The purpose of the image quality test was to evaluate the image contrast $(Q)$. The percentage contrast for hot lesions $(10,13,17$, and 22 $\mathrm{mm}$ ) and cold lesions (28 and $37 \mathrm{~mm}$ ) are defined in Equations 1 and 2, respectively. $C$ is the average count of any region and $a$ is the activity concentration, with the subindices $H$ for hot and $B$ for background for spheres of size $j$.

$$
\begin{array}{rlrl}
Q_{H, j} & =\frac{C_{H, j} / C_{B, j}-1}{a_{H} / a_{B}-1} \times 100 \%, & & \text { Eq. } 1 \\
Q_{C, j} & =\left(1-\frac{C_{C, j}}{C_{B, j}}\right) \times 100 \% . & \text { Eq. } 2
\end{array}
$$

SNRs were calculated for 7 plastic cylinders (diameters varying from 4.4 to $22.0 \mathrm{~mm}$ with volumes of $0.5-20.0 \mathrm{~mL}$ ) that were placed in an adaptation to the Ultra Delux Jaszczak Phantom manufactured in house in order to fit within a circular distribution with the larger cylinder in the center. The smaller cylinder size was closer to the spatial resolution reported by the manufacturer $(4.5 \mathrm{~mm})$. Because the injected amount of ${ }^{18} \mathrm{~F}-\mathrm{FDG}$ also depends on patient weight, activities of 350 and $200 \mathrm{MBq}$ were analyzed. Initially, the spheres were filled with ${ }^{18} \mathrm{~F}$, with an activity concen- tration of $5 \mathrm{kBq} / \mathrm{mL}$. After $83 \mathrm{~min}$, a second activity of 2.86 $\mathrm{kBq} / \mathrm{mL}$ was applied.

The SNR was defined according to Equation 3 as the ratio of the mean value of a region of interest (ROI) of $2 \times 2$ pixels (mean$R O I_{2 \times 2}$ ) minus the mean background ROI of $20 \times 20$ pixels $\left(\right.$ mean $\left._{B}\right)$ over the SD in the background $\left(S D_{B}\right)$. The SNR was calculated for gaussian filters of 1, 3, and $6 \mathrm{~mm}$ in FWHM and also for reconstructed images without any postprocessing filter method.

$$
\mathrm{SNR}=\frac{\text { mean } R O I_{2 \times 2}-\text { mean }_{B}}{S D_{B}} . \quad \text { Eq. } 3
$$

For each reconstructed series, the factors of Equation 3 were calculated, with an ROI drawn in the center of each cylinder for a total of 3 slides.

\section{Patients}

Between September 2009 and June 2011, 54 patients (14 women and 40 men; mean age, $59 \mathrm{y}$; age range, 36-84 y), with an initial diagnosis of HNSCC were referred to our Institute of Clinical Radiology and Nuclear Medicine for staging with ${ }^{18} \mathrm{~F}-\mathrm{FDG}$ PET/CT. Before the PET/CT examination, all patients had been seen clinically by an experienced ear, nose, and throat surgeon. The prospective study was approved by our hospital institutional ethic review committee. All patients who enrolled gave written informed consent for undergoing staging PET/CT before therapy. After PET/CT all patients underwent neck dissection. For anatomic correlation between imaging findings and surgery, the neck dissection specimen were divided into individual cervical lymph node levels (13). The histologic work-up of the lymph nodes served as the gold standard.

The diagnosis of HNSCC was confirmed histologically in all patients. The primary tumor locations were the base of the tongue $(n=16)$, tonsil $(n=10)$, oropharynx $(n=7)$, larynx $(n=8)$, hypopharynx $(n=4)$, and oral cavity $(n=4)$. Five patients presented with cancer of unknown primary where the tumor could not be located despite neck dissection in cervical lymph node metastases that were present.

\section{PET/CT and Image Interpretation}

Patients were imaged according to a dedicated head and neck protocol on a Biograph mCT HD PET/CT scanner with a highcounting-rate lutetium oxyorthosilicate detector (Siemens Healthcare Sector). After a fasting period of at least $6 \mathrm{~h}$, a weight-adapted intravenous injection of $250-300 \mathrm{MBq}$ of ${ }^{18} \mathrm{~F}-\mathrm{FDG}$ (ZAG Zyklotron AG) was administered. Approximately $60 \mathrm{~min}$ after tracer application, 780-mm CT using attenuation correction and a lowdose extended field of view was performed (slice thickness, $5 \mathrm{~mm}$; increment, $3 \mathrm{~mm}$ ). The anterior-posterior CT topogram was used for planning and determining the bed positions. The acquisition time for 1 bed position was 3 min. Emission images were acquired in 3-dimensional mode. After PET, high-resolution contrastenhanced biphasic CT from the skull base to the chest was performed on every patient (tube voltage, $120 \mathrm{kV}$; tube current, 50-120 mAs; slice collimation, $16 \times 1.2 \mathrm{~mm}$; pitch, 0.8 ; rotation time, $0.5 \mathrm{~s}$ ).

All studies were reconstructed into a $200 \times 200$ matrix (zoom, 1) using a resolution-recovery 3 -dimensional OSEM algorithm (3 iterative steps, 24 subsets). The postfiltering used in this study was a 3-dimensional gaussian filter. To study the effect of smoothing filter strength on diagnostic accuracy in the head and neck area, 


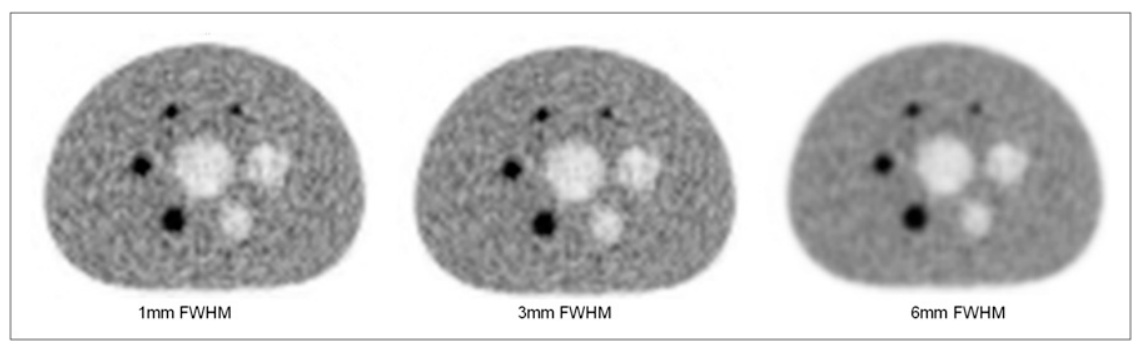

FIGURE 1. Transversal image of phantom of International Electrotechnical Commission for image quality test following NEMA protocol NU 2-2007 for different postfilters. Reconstructions were done with OSEM, 3 iterations, 24 subsets, matrix size of $200 \times$ 200 , and sphere-to-background ratio of 4:1. we used 1, 3, and $6 \mathrm{~mm}$ in FWHM as cutoffs to perform reconstructions. The head and neck, a region with low attenuation levels, requires less image postfiltering and preferably more than 2 iterations to achieve good image quality with reduced noise (10). To minimize partial-volume effect and optimize spatial resolution specifically, 1, 3, and $6 \mathrm{~mm}$ in FWHM were chosen. The average reconstruction processing time for a whole-body study was 13 min. Suggestive lymph nodes and their maximum SUV were documented on an evaluation sheet individually for the right and left sides of the neck and according to an established neck lymph node level classification (13). For determination of ${ }^{18}$ F-FDG uptake in suggestive lymph nodes, regions of interest were individually drawn around the lesions and calculated. Maximum SUV was measured and documented on the readers' sheet. Considering manufacturer-dependant hardware and software equipment for the PET/CT scanner, a maximum SUV threshold of 3.5 or less was associated with a benign lymph node not infiltrated by tumor, whereas a maximum SUV threshold of 3.5 or more was characteristic of a lymph node metastasis.

The image quality of the lymph nodes, randomly displayed in 1 , 3 , and $6 \mathrm{~mm}$ in FWHM, was evaluated visually. Two experienced independent readers performed a visual and semiquantitative analysis of the cervical lymph nodes to determine whether they were benign or malignant. Neither observer was aware of the reconstruction parameters used or the histologic findings of the lymph nodes. In the readings, all suggestive lymph nodes, including lymph nodes in the head and neck region that appeared nonmalignant, were considered. Reading was based entirely on the PET information without corresponding CT images having been viewed. Differences in reading were solved by consensus. Lymph nodes that were suggestive on PET and histologically confirmed as malignant were classified as truepositive findings. Lymph nodes that were not suggestive on PET and

TABLE 1

Phantom Study for Image Quality with 1, 3, and $6 \mathrm{~mm}$ in FWHM

\begin{tabular}{lccc}
\hline & \multicolumn{4}{c}{ Gaussian filter $(\mathrm{mm}$ in FWHM) } \\
\cline { 2 - 4 } Sphere size $(\mathrm{mm})$ & 1 & 3 & 6 \\
\hline & & & \\
10 & $32.0 \pm 4.4$ & $29.8 \pm 3.5$ & $21.1 \pm 1.6$ \\
13 & $56.8 \pm 2.6$ & $54.5 \pm 2.1$ & $42.6 \pm 0.6$ \\
17 & $63.0 \pm 4.0$ & $61.7 \pm 3.6$ & $53.2 \pm 2.1$ \\
22 & $75.0 \pm 4.9$ & $73.6 \pm 5.1$ & $64.9 \pm 4.4$ \\
28 & $64.7 \pm 1.6$ & $64.3 \pm 1.4$ & $61.3 \pm 1.4$ \\
37 & $66.8 \pm 1.4$ & $66.5 \pm 1.2$ & $64.0 \pm 1.2$ \\
\hline
\end{tabular}

Data are image contrast $(Q)(\%)$. were histologically confirmed as benign were classified as truenegative findings. Lymph nodes that were suggestive on PET but histologically confirmed as benign were classified as false-positive findings. Lymph nodes that were not suggestive on PET but were histologically confirmed as malignant were classified as falsenegative findings. Sensitivity, specificity, and statistical significance of lymph node detection and accurate diagnostic assessment at 1, 3, and $6 \mathrm{~mm}$ in FWHM was evaluated and compared, using a paired $t$ test.

\section{RESULTS}

For the phantom studies, the visual differences in image quality on the transaxial sections of the reconstructed images for different FWHM filters are displayed in Figure 1. The most apparent observation was that postfiltering at $1 \mathrm{~mm}$ in FWHM produced images of high noise, compared with the smooth images obtained at $6 \mathrm{~mm}$. The edges of the hot and cold lesions changed with filter size and were rounder with 6-mm postfiltering than with $1 \mathrm{~mm}$. The summary of the results for image quality are presented in Table 1 . The image contrast $(Q)$ was the only parameter that changed between the selected filters and was higher for 1-mm than for 3- or 6-mm postfiltering. Nevertheless, the differences between 1 and $3 \mathrm{~mm}$ were not mathematically significant for either hot and cold regions or small and big lesions. For $6 \mathrm{~mm}$, compared with $1 \mathrm{~mm}$, the difference was around $10 \%$ less for any lesion.

The results for SNR for different lesion sizes are summarized in Table 2 for each activity. The increase of the SNR was proportional to filter and region size and the applied doses. For small lesions $(4.4 \mathrm{~mm})$, the ratio between 6 and 1 $\mathrm{mm}$ in FWHM was 1.3 and 2.0 times for each activity ratio, respectively. The proportion was lower between 3 and $1 \mathrm{~mm}$ in FWHM (1.3 for $200 \mathrm{MBq}$ and 1.2 for $350 \mathrm{MBq}$ ). This proportion was also applicable to the larger studied region of $19.9 \mathrm{~mm}$. Nevertheless, the omission of postfiltering showed a better SNR than the use of 1-mm postfiltering for any region. This effect could not be demonstrated with 3-mm postfiltering.

In the 54 patients, 123 lymph nodes were evaluated on PET and histologically correlated with the neck dissection specimen. Histopathology of the dissection specimens showed 41 benign lymph nodes and 82 malignant lymph nodes (Fig. 2). The images postfiltered at 3 and $1 \mathrm{~mm}$, despite more noise, demonstrated the lymph node findings with more contrast than did the much smoother images 
TABLE 2

Results of SNR for Image Reconstruction with Activities of 200 and $350 \mathrm{MBq}$

\begin{tabular}{|c|c|c|c|c|c|c|c|c|}
\hline \multirow[b]{3}{*}{ Sphere size (mm) } & \multicolumn{8}{|c|}{ SNR } \\
\hline & \multicolumn{2}{|c|}{ All-pass* } & \multicolumn{2}{|c|}{$1 \mathrm{~mm}$ in FWHM } & \multicolumn{2}{|c|}{$3 \mathrm{~mm}$ in FWHM } & \multicolumn{2}{|c|}{$6 \mathrm{~mm}$ in FWHM } \\
\hline & $200 \mathrm{MBq}$ & $350 \mathrm{MBq}$ & $200 \mathrm{MBq}$ & $350 \mathrm{MBq}$ & $200 \mathrm{MBq}$ & $350 \mathrm{MBq}$ & $200 \mathrm{MBq}$ & $350 \mathrm{MBq}$ \\
\hline 19.9 & $16.9 \pm 0.9$ & $29.5 \pm 0.4$ & $16.0 \pm 1.0$ & $27.2 \pm 2.2$ & $18.7 \pm 0.01$ & $33.9 \pm 1.2$ & $21.8 \pm 0.2$ & $59.1 \pm 0.8$ \\
\hline 15.5 & $14.7 \pm 0.5$ & $24.9 \pm 0.4$ & $14.2 \pm 0.6$ & $24.2 \pm 1.4$ & $16.8 \pm 0.3$ & $29.0 \pm 0.5$ & $18.9 \pm 0.3$ & $48.5 \pm 1.1$ \\
\hline 12.2 & $11.3 \pm 0.7$ & $17.0 \pm 0.1$ & $10.6 \pm 0.8$ & $16.4 \pm 0.9$ & $13.1 \pm 0.2$ & $20.0 \pm 0.4$ & $14.2 \pm 0.2$ & $33.9 \pm 0.7$ \\
\hline 8.7 & $7.7 \pm 0.4$ & $10.8 \pm 0.2$ & $7.3 \pm 0.5$ & $10.6 \pm 0.6$ & $8.4 \pm 0.1$ & $12.6 \pm 0.2$ & $9.3 \pm 0.2$ & $20.5 \pm 0.6$ \\
\hline 4.4 & $2.0 \pm 0.1$ & $3.0 \pm 0.2$ & $1.9 \pm 0.1$ & $2.6 \pm 0.2$ & $2.3 \pm 0.1$ & $3.5 \pm 0.2$ & $2.6 \pm 0.1$ & $6.1 \pm 0.1$ \\
\hline
\end{tabular}

${ }^{\star}$ Reconstructed images without any postprocessing filter method.

postfiltered at $6 \mathrm{~mm}$. The impact of partial-volume effect was less in 1- and 3-mm postfiltered images than in 6-mm images. For lymph node assessment, CT information was not available for the readers. The overall image quality of the reconstructed postfiltered PET images improved from 6 $\mathrm{mm}$ in FWHM to $3 \mathrm{~mm}$ in FWHM, with significantly better lymph node visibility and contour detection despite increased noise (Fig. 3). Especially in small lymph nodes, metastatic involvement could be diagnosed more accurately

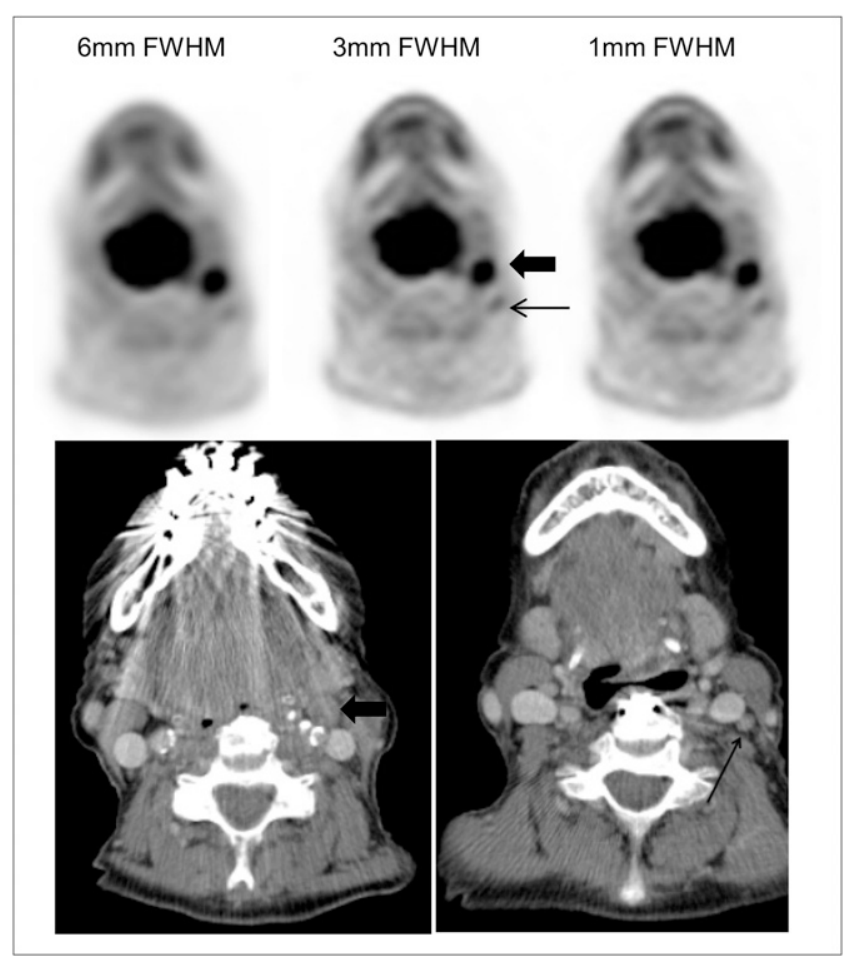

FIGURE 2. A 67-y-old woman with HNSCC of oral cavity and intensive ${ }^{18} \mathrm{~F}-\mathrm{FDG}$ uptake of primary tumor on PET. In left neck level II, 1 lymph node with intensive ${ }^{18} \mathrm{~F}-\mathrm{FDG}$ uptake (thick arrow) was histologically confirmed as lymph node metastasis anterior to left jugular vein on corresponding contrast-enhanced CT (thick arrow). Second lymph node in left neck level II with moderate ${ }^{18} \mathrm{~F}-\mathrm{FDG}$ uptake on PET (thin arrow) was confirmed benign lesion posterior and lateral to left jugular vein on CT (thin arrow). on 1- and 3-mm postfiltered images, whereas in enlarged necrotic lymph node metastases no obvious difference in image quality among 1-, 3-, and 6-mm postfiltered images could be noted (Fig. 3).

Depending on the gaussian filter applied for image reconstruction, significant differences ( $t$ test $P<0.0001$ ) in maximum SUV levels between benign and malignant lymph nodes could be demonstrated (Table 3). With decreasing size of the gaussian filter, the accuracy of lymph node characterization and SUV measurement increased, although no significant difference between 3- and 1-mm postfiltering was visible (Table 3 ). There was no overlap of the confidence intervals. They underlined the accurate distinction of maximum SUV levels in benign and malignant lymph nodes in each filter group: $6 \mathrm{~mm}$ in FWHM: benign lymph nodes, 2.7-3.1, and malignant lymph nodes, 3.7-4.6; $3 \mathrm{~mm}$ in FWHM: benign lymph nodes, 3.0-3.5, and malignant lymph nodes, 4.2-5.3; and $1 \mathrm{~mm}$ in FWHM: benign lymph nodes, 2.9-3.5, and malignant lymph nodes, 4.3-5.4.

Both readers were equally experienced in the sensitivity analysis of consensus reading. The overall sensitivities for correctly diagnosed lymph nodes were best in 3-mm postfiltered images (Table 4). With $1 \mathrm{~mm}$ in FWHM, no increase of accurate lesion detection was observed, because increasing sensitivity (95\% true-positive lymph node findings) correlated with decreasing specificity (12\% false-positive lymph node findings).

The gaussian filter-based receiver-operating-characteristic analysis of the maximum SUV levels for benign and malignant lymph nodes demonstrated statistically significant results in all 3 groups (Table 5). Postfiltering with $1 \mathrm{~mm}$ in FWHM showed the best area under the curve results and the most accurate delineation between benign and malignant lymph nodes (Table 5). Most probably because of the small number of patients, no significant differences were observed between the groups with 1 - and 3-mm postfiltering.

\section{DISCUSSION}

${ }^{18} \mathrm{~F}-\mathrm{FDG}$ PET/CT is a well-accepted diagnostic tool for lymph node staging in HNSCC, and its impact on assessment of residual or recurrent lymph node metastases in the 


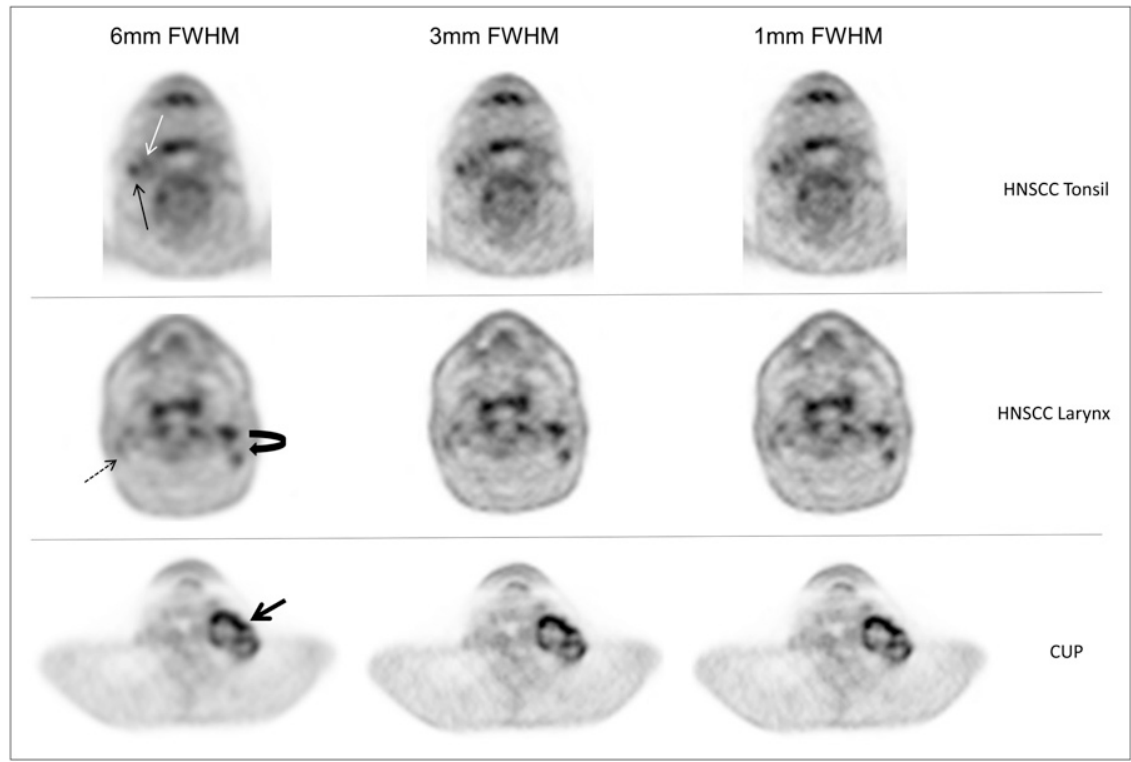

FIGURE 3. Postfiltered images at 1, 3 , and $6 \mathrm{~mm}$ in FWHM and influence of postfiltering on image quality and accurate detection of lymph node metastases. In patient with HNSCC of right tonsil, first lymph node metastasis was diagnosed correctly on all postfiltered images (thin solid black arrow), whereas second malignant node (white arrow) was misdiagnosed as false-negative finding in all postfiltered images, most probably due to small lesion size and SUV. In patient with HNSCC of larynx, benign lymph nodes could hardly be delineated on 6-mm images (dashed arrow). One- and 3-mm images enabled correct assessment of benign lymph nodes in right neck level II (dashed arrow) and metastases in left neck levels II and III (curved arrow). Diagnostic visualization of large necrotic lymph node metastases at level II on left side of neck patient with cancer of unknown primary (CUP; thick straight arrow) did not differ in any postfiltered images despite increased image blurring with $6 \mathrm{~mm}$ in FWHM.

neck after surgery and radiochemotherapy is equally high $(14,15)$. The more precise the initial staging performed, the better the expected posttherapeutic outcome for the patient. Over the last decade, technical issues in PET/CT have continuously been addressed, resulting in improved scanner technology with sensitive lesion detection and advanced data processing $(8,15,16)$. Imaging of the head and neck, an anatomically small region compared with studies of the whole body, requires dedicated imaging protocols and reconstruction algorithms to achieve sensitive and specific lesion detection (17).

In this observer study with 2 experienced readers, an OSEM algorithm was applied on a Biograph mCT scanner for PET image reconstruction in patients with an initial diagnosis of HNSCC. The intention was to analyze the effect of varying gaussian-filter strengths on image quality and sensitivity of lymph node metastasis detection. Considering that the spatial resolution of the Biograph mCT (18) is around $4.5 \mathrm{~mm}$ for the specific image reconstruction used in this study and the average size of the lymph nodes was around $10-15 \mathrm{~mm}$, we adapted standard postfiltering with 1, 3, and $6 \mathrm{~mm}$ in FWHM. In all patients, diagnostic findings could be correlated with histopathology after neck dissection.

Previously, Gutman et al. (19) have reported about the influence of iterations, subsets, and filter strengths on the diagnostic image quality of PET. Postfiltering of PET images for noise reduction has also been described but without consideration of a new scanner technology, such as established with the Biograph mCT (20-22). In our phantom study, the use of postprocessing filter methods with $1 \mathrm{~mm}$ in FWHM did not bring about an improvement in image quality. The only benefit was the increase of contrast, but the SNR was similar to images being obtained without any filter. A significant change could be noticed with the $3 \mathrm{~mm}$ in FWHM. A high contrast could be achieved, similar to that obtained with $1 \mathrm{~mm}$ in FWHM and higher than that obtained with $6 \mathrm{~mm}$ in FWHM. Also, the SNR was improved for any ROI. The application of a thicker FWHM resulted in improved SNR but reduced contrast. This effect could be confirmed during the patient study, in which $1 \mathrm{~mm}$ in FWHM did not contribute significantly to diagnostic information on PET images. These results are consistent with the publication by Akamatsu

TABLE 3

Filter-Dependant Differences in Maximum SUV Levels in Benign and Malignant Lymph Nodes

\begin{tabular}{llccrrr}
\hline Gaussian filter $(\mathrm{mm})$ & Histology & Lymph nodes & Mean maximum SUV & SD & Minimum & Maximum \\
\hline 6 & Benign & 41 & 2.89 & 0.59 & 2.2 & 5.2 \\
& Malignant & 82 & 4.20 & 1.94 & 1.8 & 11.0 \\
3 & Benign & 41 & 3.23 & 0.73 & 2.4 & 6.4 \\
& Malignant & 82 & 4.77 & 2.39 & 1.4 & 13.6 \\
1 & Benign & 41 & 3.25 & 0.81 & 1.7 & 6.8 \\
& Malignant & 82 & 4.85 & 2.43 & 2.5 & 14.6 \\
\hline
\end{tabular}


TABLE 4

Lymph Node Detection Sensitivity Analysis of Consensus Reading for 1, 3, and 6 mm in FWHM

\begin{tabular}{|c|c|c|c|c|c|c|}
\hline Gaussian filter (mm) & Histology & $\begin{array}{c}\text { No. of } \\
\text { lymph nodes }\end{array}$ & $\begin{array}{l}\text { True-positive } \\
\text { finding }\end{array}$ & $\begin{array}{l}\text { False-positive } \\
\quad \text { finding }\end{array}$ & $\begin{array}{l}\text { True-negative } \\
\text { finding }\end{array}$ & $\begin{array}{l}\text { False-negative } \\
\text { finding }\end{array}$ \\
\hline 6 & $\begin{array}{l}\text { Malignant } \\
\text { Benign }\end{array}$ & $\begin{array}{l}95 \\
28\end{array}$ & 73 & 22 & 19 & 9 \\
\hline 3 & $\begin{array}{l}\text { Malignant } \\
\text { Benign }\end{array}$ & $\begin{array}{r}112 \\
11\end{array}$ & 79 & 33 & 8 & 3 \\
\hline 1 & $\begin{array}{l}\text { Malignant } \\
\text { Benign }\end{array}$ & $\begin{array}{r}114 \\
9\end{array}$ & 78 & 36 & 5 & 4 \\
\hline
\end{tabular}

No. of total lymph nodes: 82 , malignant; 41 , benign.

(23), in which the use of a 3-mm gaussian filter was recommended for algorithms not based on a point-spread function.

In the clinical study, the best scores for lesion detection were achieved with 1 and $3 \mathrm{~mm}$ postfiltering. For each filter used, maximum SUV per lesion could be evaluated accurately, allowing distinction between potentially benign and malignant lymph nodes without overlap of the confidence intervals. Postfiltering with $3 \mathrm{~mm}$ in FWHM delivered the highest sensitivity and specificity scores for detection of lymph node metastases. The 1-mm postfiltering resulted in increased sensitivity but decreased specificity for lesion detection and therefore cannot be recommended by us for standard PET image reconstruction in the head and neck region.

Besides physical parameters that can affect PET acquisition and reconstruction, the SUV too, which is influenced by tracer kinetics, can affect image quality and interpretation $(20,22)$. Yet, physiologic parameters such as movement by the patient, inflammation, and blood glucose levels can raise maximum SUV levels and make distinction between benign and malignant lymph nodes difficult, despite advanced image postprocessing software. Therefore, despite the good diagnostic quality of PET images after postfiltering with $3 \mathrm{~mm}$ in FWHM, we cannot establish a general recommendation for image reconstruction in PET.

Komar et al. analyzed 2-dimensional and 3-dimensional ${ }^{18}$ F-FDG PET with different acquisition times (24). Acquisition time influenced uptake parameters in the patients in both modes, but surprisingly, maximum SUV levels did not differ between the 2-dimensional and 3-dimensional PET modes. In our study with fixed iterations and subsets, a significant filter-dependant difference in maximum SUV cutoff levels for benign and malignant lymph nodes was observed (Table 3). Six millimeters in FWHM clearly led to an underestimation of SUV measurements for benign and malignant lymph nodes. Three and $1 \mathrm{~mm}$ in FWHM contributed to sensitive and specific lesion detection. These findings were in line with the experience of Gutman et al. (19) and Boellard (22). PET postfiltering with a change of spatial resolution affects SUV quantification and diagnostic outcome. Postfiltering with $1 \mathrm{~mm}$ in FWHM could not increase diagnostic output significantly, because increased sensitivity for lesion detection correlated with decreased specificity. One of the probable reasons for specificity reduction due to the small postprocessing filter is related to the partial-volume effect. This effect, produced by the combination of resolution, reconstruction process, and image sampling, influences lesions of less than twice the resolution, that is, lesions of approximately $11 \mathrm{~mm}(25,26)$. In these cases, the application of correction factors as recovery coefficients is a solution, especially in oncologic imaging, but this issue was beyond the scope of the present study.

One of the limitations of this study was the fact that only PET information was available for the readers at the time of investigation. Therefore, the role of lymph node size and its correlation with the SUV with regard to benign or malignant lesion remained unconsidered in this study.

The manufacturer-dependant hardware and software of each PET scanner has to be taken into consideration. With

\section{TABLE 5}

Receiver-Operating-Characteristic Analysis of Maximum SUV Levels for Benign and Malignant Lymph Nodes for 1,3 , and $6 \mathrm{~mm}$ in FWHM

\begin{tabular}{llccc}
\hline Gaussian filter $(\mathrm{mm})$ & Histology & Mean maximum SUV & Total area under curve, $\mathrm{k}$-values & $P>\chi^{2}$ \\
\hline 6 & Benign & 2.89 & 0.76 & $P<0.0001$ \\
& Malignant & 4.2 & 0.76 & $P<0.0001$ \\
3 & Benign & 3.23 & 0.77 & $P<0.0001$ \\
& Malignant & 4.77 & 0.77 & $P<0.0001$ \\
1 & Benign & 3.25 & 0.78 & $P<0.0001$ \\
& Malignant & 4.85 & 0.78 & $P<0.0001$ \\
\hline
\end{tabular}


the integration of time-of-flight imaging in PET, a reduction of examination time and improvement of image quality due to decreased blurring can be expected (27). The characteristics of time-of-flight projections, being organized in time bins along each line of response with improved estimation of the actual image, are presently being evaluated by us in patients with HNSCC.

\section{CONCLUSION}

Compared with previous imaging protocols, for lymph node assessment on a high-resolution PET/CT scanner we consider the OSEM algorithm with 3 iterations and 24 subsets, combined with a 3-dimensional gaussian postfiltering with $3 \mathrm{~mm}$ in FWHM, optimal for lymph node staging. The continuous application of presently established PET protocols in patients with HNSCC will prove whether current acquisition and reconstruction methods are valuable and should be maintained.

\section{DISCLOSURE}

No potential conflict of interest relevant to this article was reported.

\section{REFERENCES}

1. Sadick M, Schoenberg S, Hoermann K, Sadick M. Current oncologic concepts and emerging techniques for imaging of head and neck squamous cell cancer. Laryngorhinootologie. 2012;91:S27-47.

2. Vandecaveye V, De Keyzer F, Dirix P, Lambrecht M, Nuyts S, Hermans R. Applications of diffusion-weighted magnetic resonance imaging in head and neck squamous cell carcinoma. Neuroradiology. 2010;52:773-784.

3. Yoon DY, Hwang HS, Chang SK, et al. MR, US, ${ }^{18}$ F-FDG PET/CT, and their combined use for the assessment of cervical lymph node metastases in squamous cell carcinoma of the head and neck. Eur Radiol. 2009;19:634-642.

4. Rodrigo JP, Suarez C, Ferlito A, Devaney KO, Petruzelli GJ, Rinaldo A. Potential molecular prognostic markers for lymph node metastasis in head and neck squamous cell carcinoma. Acta Otolaryngol. 2003;123:100-105.

5. Yamazaki Y, Saitoh M, Ken-ichi N, et al. Assessment of cervical lymph node metastases using FDG-PET in patients with head and neck cancer. Ann Nucl Med. 2008;22:177-184.

6. Brink I, Klenzner T, Krause T, et al. Lymph node staging in extracranial head and neck cancer with FDG PET-appropriate uptake period and size-dependence of the results. Nuklearmedizin. 2002;41:108-113.

7. Fletcher JW, Djulbegovic B, Soares HP, et al. Recommendations on the use of ${ }^{18}$ F-FDG PET in oncology. J Nucl Med. 2008;49:480-508.

8. Nahmias C, Carlson ER, Duncan LD, et al. Positron emission tomography/ computerized tomography (PET/CT) scanning for preoperative staging of pa- tients with oral/head and neck cancer. J Oral Maxillofac Surg. 2007;65: 2524-2535.

9. Schöder H, Fury M, Lee N, Kraus D. PET monitoring of therapy response in head and neck squamous cell carcinoma. $J$ Nucl Med. 2009;50:74S-88S.

10. Vogel WV, Wensing BM, van Dalen JA, Krabbe PFM, Hoogen FJA, Oyen WJG. Optimised PET reconstruction of the head and neck area: improved diagnostic accuracy. Eur J Nucl Med Mol Imaging. 2005;32:1276-1282.

11. Knoll P, Kotalova D, Köchle G, et al. Comparison of advanced iterative reconstruction methods for SPECT/CT. Z Med Phys. 2012;22:58-69.

12. National Electrical Manufacturers Association. Performance Measurements of Positron Emission Tomographs (PETs). NEMA NU 2-2007. Rosslyn, VA: NEMA; 2004.

13. Robbins KT, Clayman G, Levine PA, et al. Neck dissection classification update. Arch Otolaryngol Head Neck Surg. 2002;128:751-758.

14. Murakami R, Uozumi H, Hirai T, et al. Impact of FDG-PET/CT imaging on nodal staging for head-and-neck squamous cell carcinoma. Int $J$ Radiat Oncol Biol Phys. 2007;68:377-382.

15. Ong SC, Schöder H, Lee NY, et al. Clinical utility of ${ }^{18}$ F-FDG PET/CT in assessing the neck after concurrent chemoradiotherapy for locoregional advanced head and neck cancer. J Nucl Med. 2008;49:532-540.

16. Skretting A. A method for on-site measurements of the effective spatial resolution in PET image volumes reconstructed with OSEM and Gaussian post-filters. Radiat Prot Dosimetry. 2010;139:195-198.

17. Tarantola G, Zito F, Gerundini P. PET instrumentation and reconstruction algorithms in whole-body applications. J Nucl Med. 2003;44:756-769.

18. Biograph mCT System Specifications. Available at: www.siemens.medical.com/ siemens/de_DE/gg_nm_TBAS/files/brochures.

19. Gutman F, Gardin I, Delahaye N, et al. Optimisation of the OS-EM algorithm and comparison with FBP for image reconstruction on a dual-head camera: a phantom and a clinical ${ }^{18}$ F-FDG study. Eur J Nucl Med Mol Imaging. 2003; 30:1510-1519.

20. Casey ME. Point spread function reconstruction in PET. Siemens Molecular Imaging. 2007; www.siemens.com/mi.

21. Snyder DL, Miller MI, Thomas LJ, Politte DG. Noise and edge artifacts in maximum-likelihood reconstruction for emission tomography. J Trans Med Imaging. 1987;6:228-238.

22. Boellard R. Standards for PET image acquisition and quantitative data analysis. J Nucl Med. 2009;50:11S-20S.

23. Akamatsu G, Ishikawa K, Mitsumoto K, et al. Improvement in PET/ct image quality with a combination of point-spread function and time-of-flight in relation to reconstruction parameters. J Nucl Med. 2012;53:1716-1722.

24. Komar G, Teräs M, Seppänen M, et al. Comparison of 2D and 3D performance for FDG PET with different acquisition times in oncological patients. Nucl Med Commun. 2009;30:16-24.

25. Soret M, Bacharach SL, Buvat I. Partial-volume effect in PET tumor imaging. J Nucl Med. 2007;48:932-945.

26. Hoetjes NJ, Velden van FHP, Hoekstra OS, Hoekstra CJ, Krak NC, Lammertsma AA, Boellard R. Partial volume correction strategies for quantitative FDG PET in oncology. Eur J Nucl Med Mol Imaging. 2010;37: 1679-1687.

27. Lois C, Jakoby BW, Long MJ, et al. An assessment of the impact of incorporating Time-of-Flight (TOF) information into clinical PET/CT Imaging. J Nucl Med. 2010;51:237-245. 